\title{
EL BLOG COMO HERRAMIENTA DEL FUTURO DESARROLLO PROFESIONALEN EL GRADO DE EDUCACIÓN INFANTIL
}

\section{BLOGS AS A RESOURCE FOR THE PROFESSIONAL FUTURE IN THE EARLY CHILDHOOD EDUCATIONDEGREE}

\author{
Susana Al-Halabí1, Laura E. Gómez², Emilio López-Navarro³, Carla López- \\ Núñez ${ }^{4}$ \& Trinidad García ${ }^{5}$
}

Fecha de recepción: 03/08/2020; Fecha de revisión: 19/10/2020; Fecha de aceptación: 09/02/2021

Cómo citar este artículo:

Al-Halabí, S., Gómez, L.E., López-Navarro, E., López-Núñez, C., \& García, T. (2021). El blog como herramienta de desarrollo profesional en el Grado de Educación Infantil. Revista de Innovación y Buenas Prácticas Docentes, 10(1), 29-36.

\section{Autor de Correspondencia: alsusana@uniovi.es}

\section{Resumen:}

En la actualidad persiste un modelo de evaluación en las aulas universitarias que sigue poniendo el énfasis en el trabajo del profesorado antes que en el aprendizaje de los estudiantes. Sin embargo, estamos asistiendo a la introducción de cambios e innovaciones en los sistemas y procedimientos de evaluación que conceden un mayor protagonismo a los estudiantes. En este contexto, el objetivo de este proyecto es introducir el uso de blogs en la asignatura de "Trastornos del Comportamiento Infantil" en el Grado en Maestro de Educación Infantil de la Universidad de Oviedo, lo que favorecerá competencias relacionadas con su futuro profesional y el uso de nuevas tecnologías. En este trabajo se presentan una serie de contribuciones didácticas asociadas al uso de blogs en la educación universitaria. Tanto el profesorado como el alumnado lo valoran como un recurso útil para conformar entornos virtuales de enseñanza y aprendizaje gracias a las múltiples potencialidades que ofrece. Los alumnos informaron de una alta satisfacción con la experiencia docente y sus blogs fueron evaluados de forma muy positiva por los profesores participantes en el proyecto.

Palabras clave: aprendizaje, enseñanza y formación, innovación, tecnologías de la información y de la comunicación.

\begin{abstract}
:
Currently the evaluation framework in the University continues focusing the evaluation in the teaching staff rather than in the learning of the students. However, there are a kind of changes and innovations in the assessment systems and procedures that give a great role to students. Acco rding to this, this study aims to highlight the potential use of blogs in the active learning in the "Behavior Disorders of Childhood" subject at the University of Oviedo. This proyect will improve the students'skills related to their professional future and the use of new technologies. This work presents some contributions associated with the use of blogs in the higher education. Both teachers and students value it as a useful resource to create virtual teaching and learning environments. Students reported high satisfaction with the innovation experience. Also, their blogs were highly positively evaluated by the teachers participating in this project.
\end{abstract}

Key Words: learning, teaching and training, innovation, information and communication technologies.

\footnotetext{
' Universidad de Oviedo (España), alsusana@uniovi.es; CÓDIGO ORCID: 0000-0003-1162-2115

2 Universidad de Oviedo (España), gomezlaura@uniovi.es; CÓDIGO ORCID: 0000-0002-0776-1836

3 Universidad de Islas Baleares (España), emilio.lopez@uib.es; CÓDIGO ORCID: 0000-0002-0240-9278

${ }^{4}$ Universidad Loyola Andalucía (España), clopezn@uloyola.es; CÓDIGO ORCID: 000-0001-8897-275X

5 Universidad de Oviedo (España), garciatrinidad@uniovi.es; CÓDIGO ORCID: 0000-0001-6012-7159
} 


\section{INTRODUCCIÓN}

El uso de las TIC posibilita disponer de un volumen casi ilimitado de información que se modifica y actualiza de manera constante. Concretamente, los blogs constituyen una herramienta sencilla, fácil de implementar y muy útil a la hora de evaluar el aprendizaje del alumnado. Su uso en las aulas permite al alumnado centrarse en los contenidos, pero también en el proceso de comunicación, fomentando el aprendizaje a través de la búsqueda de información y el diálogo (Ayala, Mora, Lora, Molina \& Moyano, 2020).

Un blog es un sitio web que se actualiza periódicamente y que permite recopilar cronológicamente textos o artículos de uno o varios autores, apareciendo habitualmente primero el artículo más reciente, de forma que el autor conserva siempre la libertad de dejar publicado lo que crea pertinente. Los maestros o docentes suelen utilizar la lectura de artículos en blogs como una fuente de información constante para estar informados. A su vez, este tipo de plataforma también puede convertirse en un espacio enriqu ecedor para compartir conocimientos, experiencias y visiones de la educación. Cuando se empieza a escribir regularmente en un blog, es fácil desarrollar relaciones significativas con educadores de todo el mundo y recibir comentarios sobre nuestro trabajo dentro de ese espacio personal (Chacón \& Naranjo, 2015).

Además de los usos clásicos que como docentes podemos dar a esta herramienta, el empleo de blogs grupales no solo favorece una evaluación más sistematizada por parte del docente, sino que son una herramienta de extraordinario valor para el desarrollo de competencias de supervisión, autoevaluación y evaluación entre pares, contribuyendo a un mayor entendimiento y control del propio proceso de enseñanza-aprendizaje (Ayala et al., 2020). Así, la estructura de los blogs permite la elaboración del pensamiento de una manera secuencial y otorga un alto grado de control sobre el discurso. De esta manera, el alumno bloguero dispone de numerosas opciones que potencian el lenguaje hipertextual para componer su publicación online y contextualizar cada nuevo post dentro del discurso de su blog (Chacón \& Naranjo, 2015).

De acuerdo con la revisión de Glassman \& Kang (2016), los estudiantes suelen familiarizase pronto con los enlaces web y con las fuentes de información de otras publicaciones. Los estudios sobre la participación de los alumnos en los blogs han sugerido que a los estudiantes les gusta ver que su trabajo tiene utilidad para otras personas (Ellison \& Wu, 2008). Glassman, Bartholome \& Hur (2013) sugierenque tomar parte de la apertura de la ciencia y compartir el conocimiento es un enfoque importante e infrautilizado, y que es necesario comprender el potencial y las ramificaciones de la introducción de los blogs como parte central del proceso de enseñanza y aprendizaje.

El Espacio Europeo de Educación Superior (EEES) ha promovido en los últimos años la necesidad de establecer modelos que integren los procesos de enseñanza, aprendizaje y evaluación para facilitar la adquisición de las competencias generales y específicas de las distintas especialidades en el contexto universitario. De esta manera, la tendencia actual del EEES es incrementar el grado de participación del alumno en su proceso de enseñanza-aprendizaje (Bernabé Valero \& Blasco Magraner 2013). En este contexto, la participación del alumnado cobra tal importancia que incluso se incluye en el proceso de evaluación, tradicionalmente asignado al profesorado universitario. En la literatura científica esta cuestión es defendida por varios motivos: las ventajas de corresponsabilidad entre profesores y alumnos, las implicaciones cognitivas positivas que implica dicho proceso en el alumnado y la versatilidad y eficacia que supone el sistema de evaluación por pares y autoevaluación para el docente (Bernabé Valero \& Blasco Magraner 2013). 
Los blogs, así como la educación, son por su propia naturaleza procesos de comunicación, de socialización y de construcción de conocimiento. Una de sus características más importantes es la capacidad de interactividad, que permite que el blog pase de ser un monólogo a un diálogo en una invitación constante a la conversación (Efimova \& De Moor, 2005; Wrede, 2003). De esta forma, el alumno puede recibir el feedback de otros participantes en el debate y tomar mayor conciencia de su propio aprendizaje (Ferdig \& Trammell, 2004).

\section{DESARROLLO DE LA EXPERIENCIA DE INNOVACIÓN}

El proyecto de innovación se desarrolló en la asignatura de "Trastornos del Comportamiento Infantil", correspondiente al tercer curso del Grado en Maestro de Educación Infantil de la Universidad de Oviedo. Esta asignatura, de carácter básico, pretende que el alumno adquiera los conocimientos, competencias y actitudes necesarias para la detección y el manejo de trastornos del comportamiento que pueden surgir durante los primeros años de escolarización. El programa de la asignatura sigue una orientación psicológica que permitirá al alumno detectar, evaluar e intervenir sobre las distintas conductas problemáticas que pueden presentarse en el aula. Junto con las asignaturas "Bases Psicológicas de la Atención a la Diversidad" y "Dificultades del Aprendizaje", ofrece una visión completa de las necesidades de apoyo que el maestro de educación infantil se puede encontrar.

Los objetivos del proyecto fueron (1) potenciar nuevas metodologías de enseñanza-aprendizaje que contribuyan al desarrollo de la función docente en las que los aspectos tecnológicos no sean determinantes, y (2) desarrollar metodologías de enseñanza-aprendizaje de carácter práctico relacionado con una futura incorporación del alumnado al mundo laboral. Los indicadores del alcance de la consecución de los objetivos consistieron en una encuesta de satisfacción, la asistencia y realización de la actividad, y el criterio de los docentes implicados en la experiencia de innovación (véase apartado 3.2). Se utilizó el campus virtual de la Universidad de Oviedo como herramienta de centralización del proyecto de innovación docente (véase apartado 2.1).

Para llevar a cabo esta propuesta de innovación educativa, un total de 134 alumnos fueron informados, durante la presentación de la asignatura en el aula correspondiente de la Facultad de Formación del Profesorado, de la propuesta de elaboración de un blog en el marco de un proyecto de innovación docente aprobado por la Universidad de Oviedo. En la guía docente de la asignatura figura como tarea obligatoria la elaboración de un trabajo grupal sobre un trastorno del comportamiento infantil que debe presentarse en el aula a través de un blog. Esta actividad supuso el $40 \%$ de la nota final. Toda la información se dispuso en el campus virtual para que pudiera ser revisada por los estudiantes en cualquier momento. La revisión y difusión de los blogs contó con la supervisión y colaboración de profesores externos a la Universidad de Oviedo a modo de evaluadores externos. A continuación, se señalan los pasos realizados para la puesta en marcha de la actividad:

- Los alumnos formaron grupos de 4 a 6 personas y escogieron un tema relacionado con un comportamiento problemático infantil o un problema emocional, contextual o relacional frecuente en la infancia.

- Cada grupo elaboró un blog sobre el tema escogido de acuerdo con los apartados y contenidos establecidos en clase (a disposición de los alumnos en el campus virtual).

- Cada grupo subió su blog al campus virtual.

- Cada grupo expuso en el aula el blog y sus contenidos al resto de los compañeros.

- Cada alumno realizó una encuesta de satisf acción sobre la experiencia. 
- Los profesores de la asignatura y los colaboradores externos revisaron los blogs.

\subsection{Recursos utilizados}

El campus virtual de la Universidad de Oviedo fue la herramienta utilizada para centralizar tanto los enlaces de los blogs como la encuesta de satisfacción de los alumnos (Figura 1).

\section{¿Cómo debo enviarlo?}

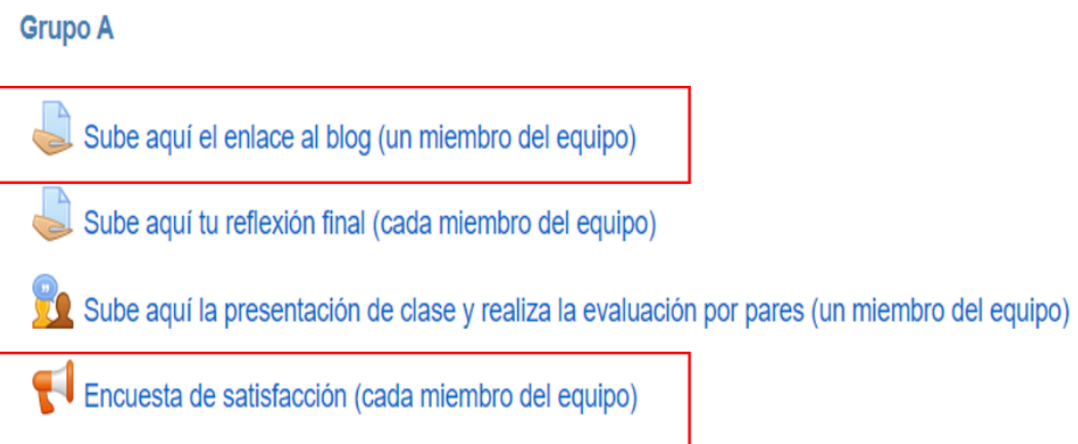

Figura 1. Recepción de los blogs del proyecto de innovación docente y encuesta de satisfacción a través del campus virtual.

Los ítems de la encuesta de satisfacción ubicada en el campus virtual pueden consultarse en la Figura 2. Las respuestas tenían un formato tipo Likert de cuatro alternativas: 1) totalmente en desacuerdo; 2) en desacuerdo; 3) de acuerdo; 4) totalmente de acuerdo.

\begin{tabular}{ll}
\hline Ítem 1 & $\begin{array}{l}\text { Creo que la realización del trabajo grupal ha contribuido a mejorar mi formación } \\
\text { sobre el tema elegido. }\end{array}$ \\
\hline Ítem 2 & $\begin{array}{l}\text { Las temáticas propuestas para los trabajos g rupales son interesantes para mi } \\
\text { futura práctica profesional. }\end{array}$ \\
\hline Ítem 3 & $\begin{array}{l}\text { Con la realización del blog he contribuido a la diseminación de contenidos } \\
\text { interesantes para los/as maestros/as de educación infantil. }\end{array}$ \\
\hline Ítem 4 & $\begin{array}{l}\text { La exposición de los blogs y los distintos trabajos en clase ha despertado mi } \\
\text { curiosidad sobre otros temas interesantes para mi futura práctica docente. }\end{array}$ \\
\hline Ítem 5 & $\begin{array}{l}\text { Los temas de los trabajos complementan los temas del programa docente de la } \\
\text { asignatura. }\end{array}$ \\
\hline Ítem 6 & $\begin{array}{l}\text { La realización del trabajo grupal me ha permitido mejorar mis habilidades de } \\
\text { trabajo en equipo. }\end{array}$ \\
\hline
\end{tabular}

Figura 2. Encuesta de satisfacción

Fuente: Elaboración propia.

Asimismo, se puso a disposición de los alumnos varios links para elaborar blogs de manera gratuita (Figura 3). 


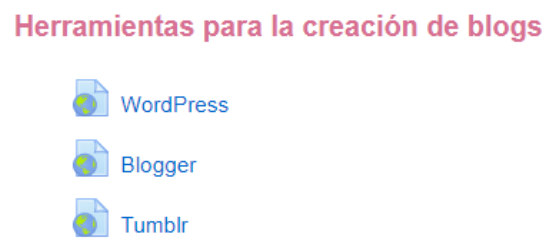

Figura 3. Recursos de blogs disponibles en el campus virtual de la asignatura.

\section{RESULTADOS}

Se presentan los resultados del proyecto de innovación atendiendo a tres criterios: los blogs realizados por los alumnos, los indicadores de resultados de la Universidad de Oviedo y las respuestas de los alumnos a la encuesta de satisfacción con la experiencia.

\subsection{Blogs realizados por los alumnos}

A continuación, se presentan los enlaces a los blogs realizados por los alumnos:

- https://guia-para-maestros-as-sobre-el-tea.webnode.es/

- https://obsesivocompulsivouniovi.blogspot.com

- https://acosoybullyingsocial.family.blog/

- https://wordpress.com/posts/trastornodeansiedad771308192.wordpress.com

- https://guia-para-maestros-as-sobre-el-tea.webnode.es/

- https://trastornosconductasocial.family.blog/home/

- https://elmaltratoinfantiluniovi.blogspot.com

- https://guiatdah2020.webnode.es/

- https://santrabadela.wixsite.com/misitio

- https://y0tengountic. blogspot.com/

- https://elmundochiquitin.blogspot.com/

- https://problemas-del-sueno9.webnode.es/

\subsection{Indicadores de resultados}

En la Figura 4 se pueden consultar los indicadores de resultados establecidos en el proyecto de innovación docente aprobado por el Centro de Innovación de la Universidad de Oviedo.

\begin{tabular}{lcl}
\hline Indicador & Modo de evaluación & Rangos fijados y obtenidos \\
\hline $\begin{array}{l}\text { Encuesta elaborada ad hoc por los } \\
\text { profesores implicadosen este proyecto } \\
\text { que contendrá ítems relacionados con } \\
\text { la satisfacción de los estudiantes en los } \\
\text { aspectos previamente señalados. }\end{array}$ & $\begin{array}{c}\text { Encuesta en el campus } \\
\text { virtual }\end{array}$ & \multicolumn{1}{c}{ Satisfacción alta } \\
\hline $\begin{array}{l}\text { Aumento de la asistencia a las clases } \\
\text { presenciales y las tutorías. }\end{array}$ & Asistencia clase & $\begin{array}{l}\text { La asistencia a clase fue alta y el 98\% } \\
\text { de los alumnos se presentaron al } \\
\text { examen de la asignatura. }\end{array}$ \\
\hline Calidad del blog y los trabajos & $\begin{array}{c}\text { Criterio externo y de los } \\
\text { docentes de la } \\
\text { asignatura }\end{array}$ & $\begin{array}{l}\text { Todos los alumnos superaron el } \\
\text { punto de corte necesario para } \\
\text { aprobar el trabajo del proyecto de } \\
\text { innovación. En dicha nota se incluyó } \\
\text { el criterio de fiabilidad externos de los } \\
\text { colaboradores del proyecto. }\end{array}$ \\
\hline
\end{tabular}

Figura 4. Valoración global de los indicadores de resultados.

Fuente: Elaboración propia 


\subsection{Encuesta de satisfacción}

La Figura 5 recoge los datos de las respuestas de los alumnos participantes en el proyecto de innovación.

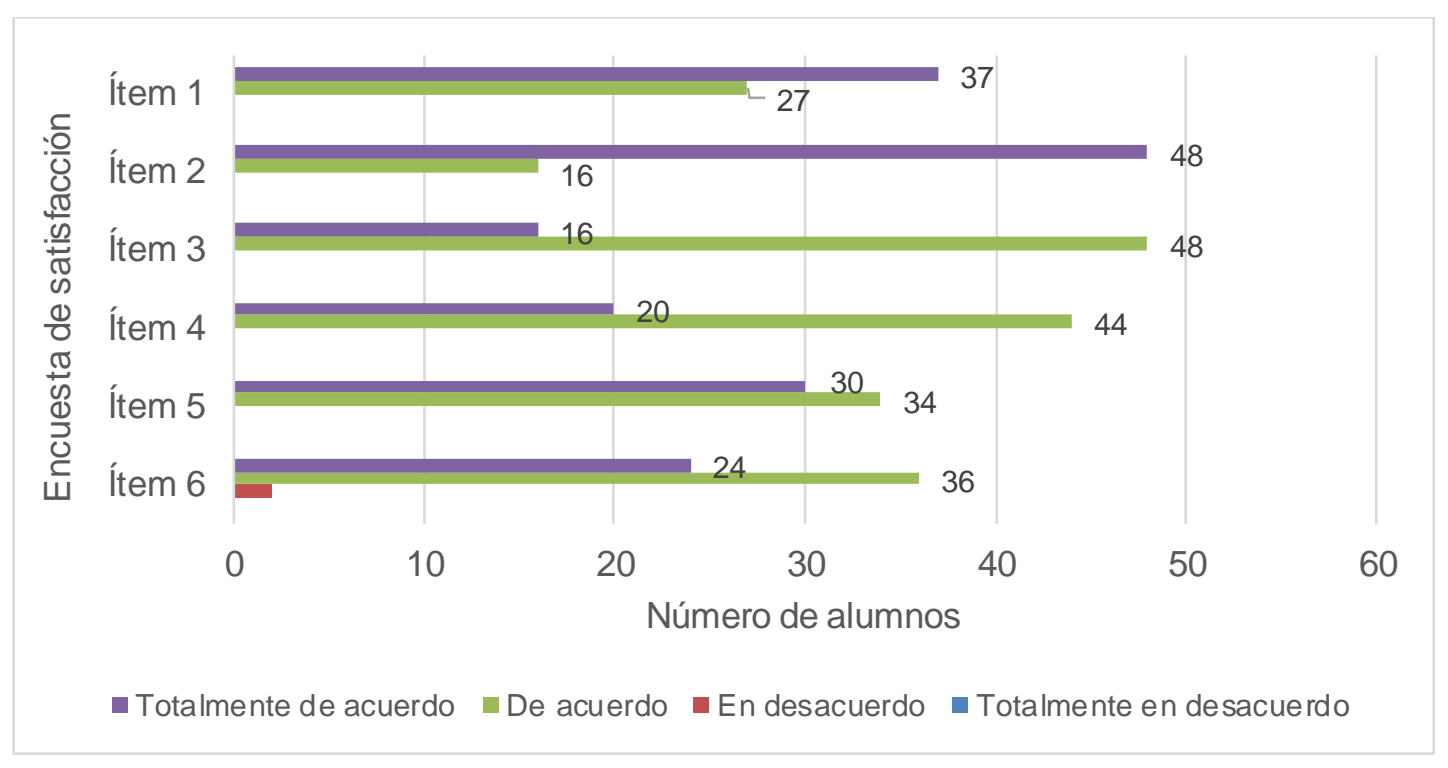

Figura 5. Respuestas de los alumnos a la encuesta general de satisfacción con el proyecto de innovación docente.

\section{CONCLUSIONES}

En la actualidad, existe un interés pedagógico por transformar las tecnologías de la información y comunicación en tecnologías para el aprendizaje y el conocimiento (Molina Aventosa, Valenciano Valcárcel \& Valencia-Peris, 2015). En este trabajo se presentan una serie de contribuciones didácticas asociadas al uso de blogs en la Educación Superior a partir de una experiencia de innovación llevada a cabo en el Grado de Educación Infantil de la Universidad de Oviedo por un grupo de profesores universitarios durante el curso académico 2019/2020.

Tanto el profesorado como el alumnado participante en las asignaturas donde se ha puesto en práctica el uso de blogs, lo valoran como un recurso útil para conformar entornos virtuales de enseñanza y aprendizaje gracias a las múltiples potencialidades que ofrece. Entre ellas se destacan: facilitar el acceso al conocimiento, promover una participación más activa y reflexiva en la construcción de los aprendizajes, amplificar la experiencia social del aprendizaje, proporcionar evidencias sobre el progreso de los estudiantes que ayuden a reorientar el proceso de enseñanza-aprendizaje y estimular el juicio crítico (Molina Aventosa et al., 2015).

Así, de acuerdo con la literatura científica y las respuestas de los alumnos a la encuestade satisfacción podemos señalar que la contribución didáctica de la innovación aplicada consta de los siguientes aspectos:

- El uso de blogs mejora de los procesos y productos del aprendizaje: la participación en actividades con los compañeros es considerada como una importante experiencia educativa que contribuye a la notable mejora del aprendizaje y al desempeño del futuro rol profesional como maestros.

- Incremento del aprendizaje y el rendimiento: la realización de los trabajos sirve para profundizar en temas centrales de la asignatura. 
- Se convierte en un incentivo para mejorar el trabajo grupal y el propio esfuerzo: la implicación del alumnado aumenta cuando el contenido de sus trabajos es público.

- Estimula el pensamiento y el aprendizaje crítico.

- Importancia para el desarrollo de la carrera profesional: la implicación en actividades abiertas a la comunidad educativa es valorada por los propios estudiantes como un elemento clave para el desarrollo de la carrera profesional.

- Desarrollo de competencias en el uso responsable del Blog y de su dimensión como recursos al servicio de la comunidad educativa.

Igualmente, de acuerdo con Glassman y Kang (2016), los estudiantes se han familiarizado rápido con la elaboración de los blogs, a pesar de la inmensa mayoría nunca los había hecho con anterioridad. Los alumnos han mostrado una gran satisfacción con el trabajo realizado, lo cual queda patente a través del propio criterio de los docentes y de las respuestas de los alumnos a la encuesta de satisfacción. Los resultados están en la línea del trabajo de Kamel Boulos, Maramba y Wheeler (2006) que afirmaron que, si se implementan de manera efectiva, los wikis, blogs y podcasts podrían of recer una manera de mejorar experiencias de aprendizaje de los alumnos universitario, así como su compromiso con determinados contenidos.

Tras el desarrollo del proyecto de innovación, podemos señalar que la principal fortaleza radica en la elevada satisfacción por parte de los alumnos, la buena ejecución del trabajo y la valoración positiva sobre la utilidad para la práctica profesional. Asimismo, se ha dotado a la comunidad educativa de material concreto sobre trastornos del comportamiento en el aula, lo que puede derivar en un servicio de conocimiento aplicado a la sociedad y los profesionales. No obstante, como limitación, podemos señalar que seríainteresante conocer si los diversos blogs creados en la asignatura han llegado a otros miembros de la comunidad educativa, así como tener criterios de comparación con otros blogs del mismo campo de conocimiento. También sería interesante incluir el feedbackoral de los compañeros de clase acerca de los contenidos como parte de las competencias necesarias para el ejercicio profesional de los futuros maestros. Dicha modificación ya ha sido solicitada y aprobada por la Universidad de Oviedo en el presente curso académico.

Finalmente, para concluir, cabe señalar que los maestros del ciclo de educación infantil suelen ser responsables de un aula de niños de la misma edad, pero con capacidades e intereses muy distintos, de ahí la necesidad de adquirir capacidad de análisis y de establecer criterios solidos de evaluación. Entre sus funciones destacan promover el aprendizaje en la primera infancia, facilitar la evolución del lenguaje y escrito, identificar las habilidades, intereses y necesidades especiales del alumnado, fomentar la buena convivencia en el aula y la adquisición de hábitos. Además, todas estas funciones las llevan a cabo en centros educativos que suelen contar con personal de muy diversa cualificación, dependiendo de las necesidades que presenten los alumnos escolarizados en cada centro. Por lo tanto, se hace imprescindible desarrollar habilidades relacionadas con el trabajo en equipo y la capacidad de consenso. Estas habilidades pueden entrenarse a través del uso de blogs grupales como componente de los contenidos de las asignaturas del Grado en Educación Infantil.

\section{REFERENCIAS}

Ayala, N., Mora, R., Lora, A.J., Molina, A. M., \& Moyano, M. R. (2020). Desarrollo e implementación de un blog colaborativo como instrumento de coevaluación en el Grado de Veterinaria. Revista de Innovación y Buenas Prácticas Docentes, 9, 6170. DOI: https://doi.org/10.21071/ripadoc.v9i1.12610 
Bernabé Valero, G., \& Blasco Magraner, J. S. (2013). Evaluación por pares y autoevaluación en el aula universitaria: una visión desde el enfoque por competencias. Recuperado de http://web.ua.es/es/ice/jornadasredes/documentos/2013- posters/335209.pdf

Chacón Almeda, R., \& Naranjo Ramírez, F. (2015). La comunicación en las TIC aplicadas al aula. Madrid, España: Fundación UNED.

Efimova, L., \& De Moor, A. (2005). Beyond personal webpublishing: An exploratory study of conversational blogging practices. En Proceedings of the Thirty-Eighth Hawaii International Conference on System Sciences (HICSS-38). Recuperado de https://doc.telin.nl/dscgi/ds.py/Get/File-44480/

Ellison, N., \& Wu, Y. (2008). Blogging in the classroom: A preliminary exploration of student attitudes and impact on comprehension. Journal of Educational Multimedia and Hypermedia, 17, 99-122.

Glassman, M., Bartholomew, M., \& Hur, E. H. (2013). The importance of the second loop in educational technology: An action science study of introducing blogging in a course curriculum. Action Research, 11, 337-353. DOI: http://dx.doi.org/10.1177/1476750313502555

Glassman M., \& Kang, M. J. (2016). Teaching and learning through open source educative processes. Teaching and Teacher Education, 60, 281-290. DOI: http://dx.doi.org/10.1016/j.tate.2016.09.002

Kamel Boulos, M.N, Maramba, I., \& Wheeler, S. (2006). Wikis, blogs and podcasts: a new generation of Web-based tools for virtual collaborative clinical practice and education. BMC Medical Education, 6, 41. DOI: http://dx.doi.org/10.1186/14726920-6-41

Molina Aventosa, P., Valenciano Valcárcel, J., \& Valencia-Peris, A. (2015). Los blogs como entornos virtuales de enseñanza y aprendizaje en Educación Superior. Revista Complutense De Educación, 26, 15-31. DOI: https://doi.org/10.5209/rev_RCED.2015.v26.43791 